\title{
eSPC, an Online Data Analysis Platform for Molecular Biophysics
}

\author{
Maria m. Garcia Alai
}

EMBL, Hamburg, Germany

garcia@embl-hamburg.de

\begin{abstract}
All biological processes rely on the formation of protein-ligand, protein-peptide and proteinprotein complexes. Studying the affinity, kinetics and thermodynamics of binding between these pairs is critical for understanding basic cellular mechanisms. There are many different technologies designed for probing interactions between biomolecules, each based on measuring different signals (fluorescence, heat, thermophoresis, scattering and interference; among others). Evaluation of the data from the binding experiments and its fitting is an essential step towards the quantification of binding affinities. Here, we present user-friendly online tools to analyze biophysical data from steady-state fluorescence spectroscopy, microscale thermophoresis and differential scanning fluorimetry experiments. The modules from our data analysis platform (spc.embl-hamburg.de) contain classical thermodynamic models and clear user guidelines for the determination of equilibrium dissociation constants $\left(\mathrm{K}_{d} \mathrm{~S}\right)$ and thermal unfolding parameters such as melting temperatures $\left(\mathrm{T}_{\mathrm{m}} \mathrm{s}\right)$.
\end{abstract}

Keywords: online server, open science, molecular interactions, binding affinity, $K_{d} s$, microscale thermophoresis, differential scanning fluorimetry, protein stability, $\mathrm{Tm}$, ligand screening 